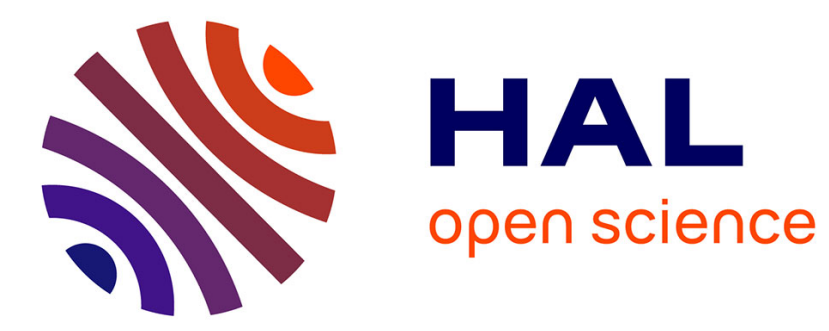

\title{
Dynamics of a grain on a sandpile model
}

\author{
L. Quartier, Bruno Andreotti, Stéphane Douady, A. Daerr
}

\section{To cite this version:}

L. Quartier, Bruno Andreotti, Stéphane Douady, A. Daerr. Dynamics of a grain on a sandpile model. Physical Review E , 2000, 62 (6), pp.8299-8307. 10.1103/PhysRevE.62.8299 . hal-01591074

\section{HAL Id: hal-01591074 https://hal.science/hal-01591074}

Submitted on 20 Sep 2017

HAL is a multi-disciplinary open access archive for the deposit and dissemination of scientific research documents, whether they are published or not. The documents may come from teaching and research institutions in France or abroad, or from public or private research centers.
L'archive ouverte pluridisciplinaire HAL, est destinée au dépôt et à la diffusion de documents scientifiques de niveau recherche, publiés ou non, émanant des établissements d'enseignement et de recherche français ou étrangers, des laboratoires publics ou privés. 


\title{
Dynamics of a grain on a sandpile model
}

\author{
L. Quartier, B. Andreotti, S. Douady, and A. Daerr \\ Laboratoire de Physique Statistique de l'ENS, 24 rue Lhomond, 75231 Paris Cedex 05, France
}

(Received 5 June 2000)

\begin{abstract}
The dynamics of a macroscopic grain rolling on an inclined plane composed of fixed identical grains is investigated both experimentally and theoretically. As real sand, the system exhibits an hysteretic transition between static and dynamical states: for angles smaller than $\varphi_{d}$, the roller always stops, for angles larger than $\varphi_{s}$, it spontaneously starts rolling down. But for angles between $\varphi_{d}$ and $\varphi_{s}$, it can be either at rest or in motion with a constant velocity. It is shown that the limit velocity is given by the equilibrium between gravity driving and dissipation by the shocks. Moreover, the rough plane acts as a periodic potential trap whose width and depth decrease when the angle is increased: the static angle $\varphi_{s}$ corresponds to the angle for which the trap disappears; the dynamical angle $\varphi_{d}$ to that for which the limit velocity is sufficient to escape from the trap. Finally, a continuous description of the force globally acting on the grain is proposed, which preserves this hysteretic behavior.
\end{abstract}

PACS number(s): 83.70.Fn, 45.05.+x

\section{INTRODUCTION}

Although sand beaches provide a wide playing field that allows many pleasant experiments, granular media remains closed to an intuitive understanding. There are mainly two reasons for that. One is related to the difficulty of describing granular media, the other to the lack of physical mechanisms that would simply explain its main behaviors.

First particularity, a sandpile is able to remain static even with an inclined free surface. This equilibrium is generally characterized by the free-surface slope $\tan \varphi$, following the analogy with solid friction [1], besides not more intuitive. After having built dynamically a sandpile, it remains static with an angle of repose $\varphi_{d}$. This means that any motion on the surface spontaneously stops if $\varphi<\varphi_{d}$. This sandpile at rest can now be inclined up to a second critical angle $\varphi_{s}$ at which an avalanche occurs. $\varphi_{s}$ is thus the maximum angle of stability: for $\varphi>\varphi_{s}>\varphi_{d}$, the sandpile spontaneously starts flowing. Also the dynamics of avalanches presents original features: contrary to a liquid, only a thin layer of grains is set into motion and contrary to a solid, the avalanche does not accelerate indefinitely, and reaches a finite limit velocity.

The transition between solid and liquidlike behaviors is hysteretic: when the free surface is between $\varphi_{d}$ and $\varphi_{s}$ the sandpile can be either flowing or at equilibrium, depending on the history. In other words, the free-surface slope is not sufficient to describe the state of the system and its evolution, the fact that it is in motion or not being crucial. Actually, the situation is even worse since both the starting angle $\varphi_{s}$ and the dynamical angle $\varphi_{d}$ depend not only on the material but also on the microscopic arrangement of grains, on the preparation of the sandpile, on its structure [2], etc.

The second particularity is the absence of a mesoscopic lengthscale between the grain diameter $d$ and the macroscopic scale (typically $10^{2} d-10^{4} d$ ) at which the local properties could be averaged. Because the number of grains is always too large to consider them individually, an important problem is the possibility of describing granular material as a continuous system. We are thus interested in two problems: (1) What are the basic mechanisms at the scale of one grain that govern the dynamics of a sandpile? and (2) Can we build a continuous "macroscopic" model to describe these dynamics?

In order to get an intuive idea of the physical mechanisms acting in such a complex system, we will investigate the previous questions in the simpler case of a single "enlarged" grain on a rough inclined plane (the rest of the sandpile). This study has been previously developed [3-10] with the idea (among others) of studying the segregation phenomenon. The dynamics of a single bead was thus studied, varying the plane roughness, i.e., the bead size compared to the size of bumps beneath it: at large angle (and large size), the bead jumps and accelerates indefinitely, for smaller angles (and smaller size) the bead stabilizes on a constant velocity, and below a threshold value it stops. We already see that this simple system exhibits a bifurcation between static and flowing behaviors. Our aim is to reexamine this experiment, focusing not only on the mean velocity, but also on transients (on the effective friction force acting on the bead) and on the subcritical transition between static equilibrium and motion.

The experimental setup is described in Sec. II. In the next part, the stationary states are characterized as a function of the slope for different materials and the global friction force is determined experimentally. A simple discrete model is proposed in Sec. IV and compared to the experimental results. The possibility of a continuous description of the forces acting on one grain is finally discussed.

\section{EXPERIMENTAL SETUP}

\section{A. Setup}

The experiment consists in studying the motion of a brass cylinder of diameter $d=23 \mathrm{~mm}$ on an inclined plane made by an array of 32 cylinders identical to the rolling one (Fig. 1). The cylindrical geometry, although leading to imperfect contacts, was chosen to enforce the two-dimensional stability of the motion and to avoid the friction on possible lateral walls. The plane ( $800 \mathrm{~mm}$ long and $60 \mathrm{~mm}$ wide) can be inclined at a variable angle $\varphi$ (Fig. 1). The distance $D$ between two neighboring cylinders is directly linked to the compacity of 


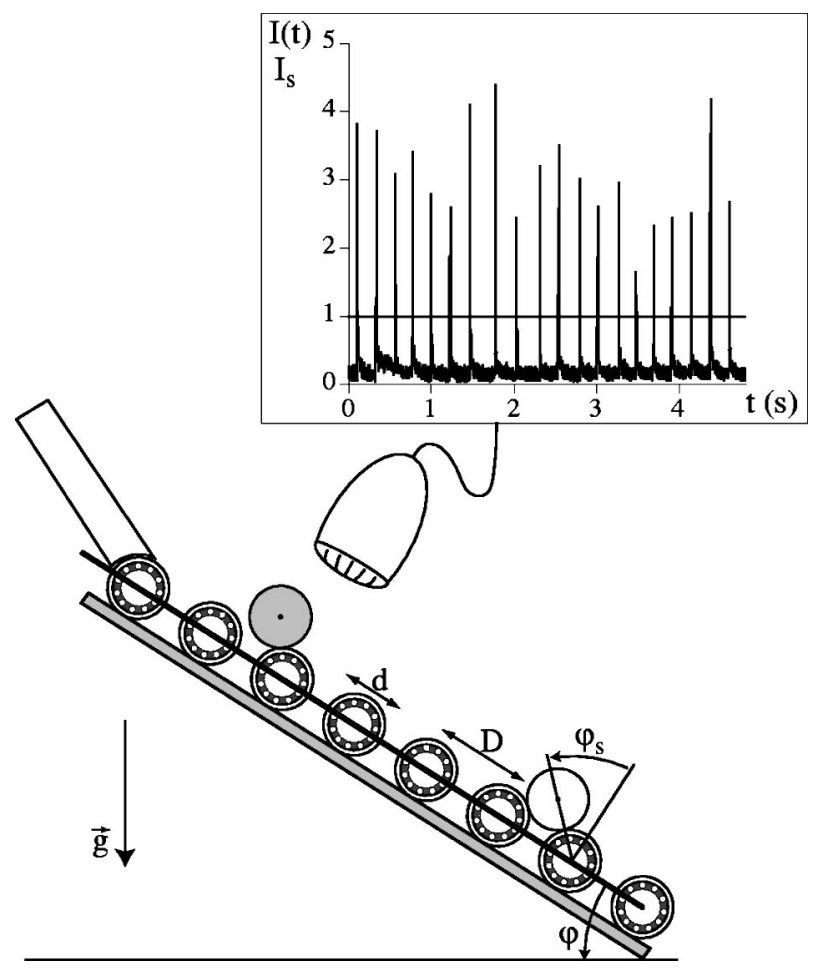

FIG. 1. Schematic view of the experiment. A cylinder of diameter $d$ is rolling on a rough plane made by an array of fixed identical cylinders. The sound emitted during the roller's motion is recorded and analyzed to determine when each shock has occurred.

the plane and is equal to $25 \mathrm{~mm}$ for all the results presented here. In order to vary (and control) the initial velocity of the roller, it is released with a null velocity on a "launching pad"' at various heights.

We used two methods to determine the cylinder velocity. A video camera was used to measure the position as a function of time (Figs. 2 and 4). This method is however limited by the resolution in time $(50 \mathrm{~Hz})$ and space. We thus took benefit from the particularity of the system: during the motion of the roller, it collides with the cylinders beneath it. To perform measurements, we simply record the sound emitted

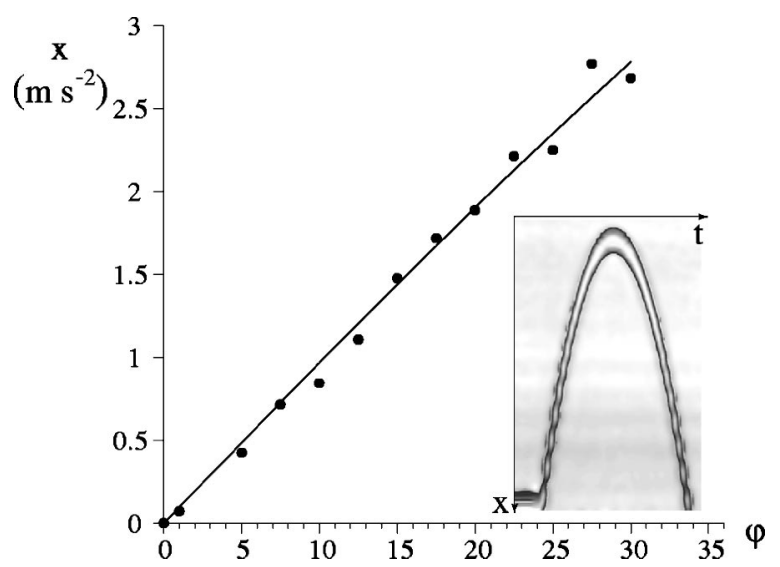

FIG. 2. Motion of the roller on a smooth plane. Main graph: acceleration $\ddot{x}$ as a function of the plane angle $\varphi$. The slope is directly related to the dimensionless moment of inertia $j$. Inset: spatiotemporal trajectory obtained by plotting one CCD line parallel to the plane as a function of time.
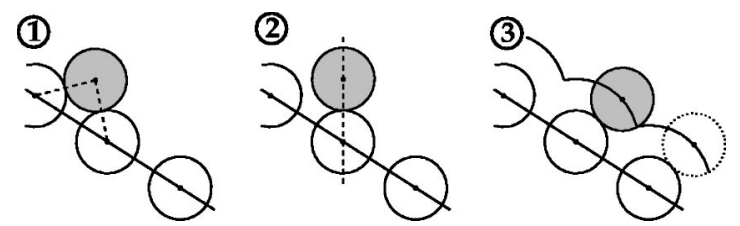

FIG. 3. The possible stationary states are (1) the equilibrium of the roller trapped between the cylinders beneath it, (2) the unstable equilibrium of the roller on top of the cylinder, and (3) the periodic motion of the roller.

by the successive shocks (Fig. 1) using the $22 \mathrm{kHz}$ audio input of our computer. After a simple thresholding, the successive times of collision $T_{n}$, numbered by the index $n$, can be determined within a $0.5 \mathrm{~ms}$ error. The mean velocity of the moving cylinder between two successive shocks $n$ and $n+1$ is parallel to the inclined plane and equal in modulus to

$$
\bar{V}_{n}=\frac{D}{T_{n+1}-T_{n}} .
$$

Since all the quantities can be properly scaled using the roller's weight $m \simeq 110 \mathrm{~g}$ its diameter $d$, and gravity $g$, the experiment for a given geometry is controlled only by the plane angle $\varphi$.

\section{B. Moment of inertia}

As the cylinder rolls on the ones underneath, it is important to determine its moment of inertia $J=j m d^{2} / 4$ about its axis. To measure the dimensionless moment of inertia $j$, the roller is thrown upward onto a smooth plane made of brass (Fig. 2). It then rolls up, stops, rolls down and, as a first approximation, does not slide on the plane. The spatiotemporal trajectory appears to be parabolic (Fig. 2), the acceleration being thus constant. The acceleration is plotted as a function of the plane angle $\varphi$ in Fig. 2. It is proportional to the component of gravity parallel to the plane $g \sin \varphi$.

If we assume that there is strictly no sliding, the roller velocity $\dot{x}$ is related to its angular velocity $\dot{\theta}$ by $d \dot{\theta} / 2$. If we furthermore assume that the contact between the plane and the roller is perfectly linear, the moment of the reaction force about this line is null. The moment of the force of gravity about the contact line is $K=1 / 2 m g d \sin \varphi$ and the angular momentum is simply $M=1 / 4(1+j) m d^{2} \dot{\theta}$. From the equation of motion $\dot{M}=K$, we derive the expression of the acceleration $\ddot{x}=g \sin \varphi /(1+j)$.

From the fit of experimental data (Fig. 2), we deduce the dimensionless moment of inertia $j=0.76$. As expected, this value is between $j=0.5$ and $j=1$, which correspond, respectively, to the case of a tube and to the case of a full cylinder.

\section{EXPERIMENTAL RESULTS}

\section{A. Stationary states}

The simplest stationary state that can be observed is the equilibrium of the roller in the trap created by two cylinders [situation (1) in Fig. 3]. There exists a second equilibrium position [situation (2) in Fig. 3] when the roller is straight up over the cylinder beneath it, but it is obviously unstable. 


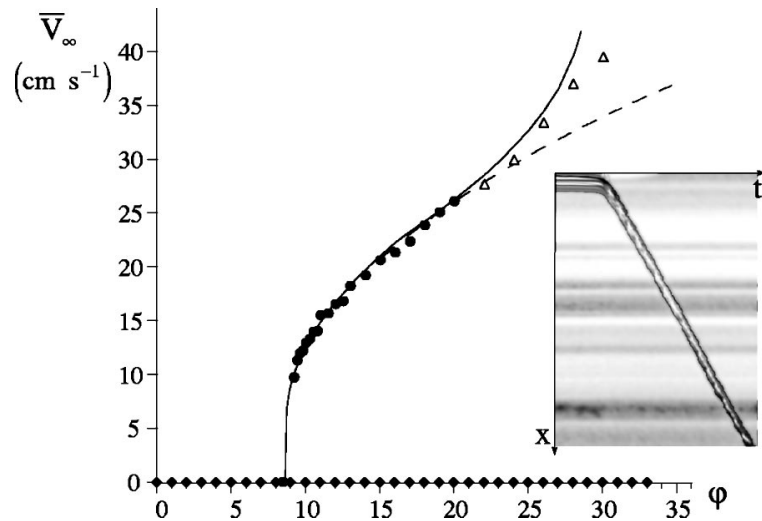

FIG. 4. Limit velocity as a function of the plane angle. The equilibrium (black diamonds) is observed up to the static angle $\varphi_{s}$. Above the dynamical angle $\varphi_{d}$, the roller can reach a nonzero limit velocity (black circles), before it starts taking off the cylinders beneath it (triangles). The fit by the microscopic model assuming permanent contact is shown in the solid-dashed line, and the model including the possibility of takeoff and free flight is shown in the solid line. Inset: spatiotemporal trajectory obtained by plotting one video line parallel to the plane as a function of time.

When we increase the angle $\varphi$ above a critical angle $\varphi_{s}$ $\simeq 33^{\circ}$ (the static angle), the cylinder spontaneously starts rolling down the plane. This critical angle $\varphi_{s}$ corresponds to the position of the plane for which the line joining the center of the roller to the center of the underneath cylinder is vertical [collapse of situations (1) and (2) of Fig. 3)]. In other words, at $\varphi=\varphi_{s}$, the potential trap disappears. $\varphi_{s}$ can thus be derived from pure geometrical considerations. Using Fig. 1 , we immediately obtain the relation:

$$
\sin \varphi_{s}=\frac{D}{2 d}
$$

which gives a numerical value close to the measured one $\left(\simeq 33^{\circ}\right)$.

If we now give an initial impulse to the roller at rest (for $\varphi<\varphi_{s}$, see above), two different behaviors are observed. At small angle, it quickly stops even when it is set into motion with a large kinetic energy. The only stationary state is thus the static one. In an intermediate angle range, the position of equilibrium is stable towards small disturbances but, if a sufficiently large initial velocity is given, the cylinder goes on rolling and reaches a constant mean velocity $\bar{V}_{\infty}$ (inset of Fig. 4). This is the third possible stationary state [situation (2) on fig. 3]. This constant velocity regime can be reached above the dynamical angle $\varphi_{d} \simeq 8.5^{\circ}$ which is far below the static one $\varphi_{s}$. This difference between $\varphi_{d}$ and $\varphi_{s}$ shows that there is a large hysteresis between static and rolling states.

To summarize, there exists for $\varphi_{d}<\varphi$ a stationary regime that is reached after a short transient. This means that the local mean velocity $\bar{V}_{n}$ becomes independant of the shock number $n\left(\bar{V}_{n}=\bar{V}_{\infty}\right)$. The other stable stationary state is the equilibrium of the roller in the trap created by the two neighboring cylinders $\left(\bar{V}_{\infty}=0\right)$. It is stable for $\varphi<\varphi_{d}$, metastable for $\varphi_{d}<\varphi<\varphi_{s}$, and does not exist any more for $\varphi_{s}<\varphi$. The transition between equilibrium and motion is thus subcritical.
To characterize these stationary states, we measured the limit mean velocity $\bar{V}_{\infty}$ for different angles $\varphi$. For this, we averaged $\bar{V}_{n}$ over several shocks (typically 10) in the stationary regime. The resulting curve is shown in Fig. 4. The black diamonds show the angles for which the equilibrium state $\left(\bar{V}_{\infty}=0\right)$ is observed; the last point gives the static angle $\varphi_{s} \simeq 33^{\circ}$. The black circles correspond to the constant velocity regime. The first point has been drawn at the angle for which this regime disappears, i.e., at the dynamical angle $\varphi_{d} \simeq 8.5^{\circ}$. We can observe that the limit velocity increases very quickly above the bifurcation threshold: in particular we did not get any measurement below $10 \mathrm{~cm} \mathrm{~s}^{-1}$ (one-fourth of the total range). The triangles correspond to the points for which the roller is no more in permanent contact with the plane. At last, the solid and dashed lines correspond to the microscopic model developed in the next section. The soliddashed line is obtained assuming a permanent contact between the roller and the rough plane and the solid line to the full model, including takeoffs of the roller at large angles $\varphi$. It can now be seen that the model provides a very good fit of the data.

To investigate the influence of the material, we have covered the roller by a thin felt layer, which has a larger friction coefficient than brass (Fig. 5, top) and by a rubber layer, which is more elastic than brass (Fig. 5, middle). It turns out that the material properties do not affect strongly the results. This means that the energy dissipation essentially comes from the shocks and not from the solid friction between the roller and the plane. We also investigated the case where the cylinders composing the inclined plane are let free to turn around their axis (Fig. 5, bottom). In that case, the dynamical angle is larger than previously $\left(\varphi_{d} \simeq 11.7^{\circ}\right)$. Essentially, this comes from the fact that the rotation kinetic energy is no longer transferred into translation motion when a shock occurs. This shows the importance of rotation in the problem.

\section{B. Global forces during transients}

The stationary states being characterized, we can investigate the force globally acting on the roller. For this, we measured experimentally the acceleration during transients. Assuming that the acceleration is constant between the shocks $n-1$ and $n+1$, the distance covered by the roller depends quadratically on time so that we get two relations:

$$
\begin{aligned}
& D=\bar{V}\left(T_{n+1}-T_{n}\right)+\frac{1}{2} \frac{\delta \bar{V}}{\delta t}\left(T_{n+1}-T_{n}\right)^{2}, \\
& -D=\bar{V}\left(T_{n-1}-T_{n}\right)+\frac{1}{2} \frac{\delta \bar{V}}{\delta t}\left(T_{n-1}-T_{n}\right)^{2} .
\end{aligned}
$$

The acceleration can thus be deduced from the successive times $T_{n}$ at which the shocks occur:

$$
\frac{\delta \bar{V}}{\delta t}=\frac{2 D\left(2 T_{n}-T_{n-1}-T_{n+1}\right)}{\left(T_{n+1}-T_{n}\right)\left(T_{n}-T_{n-1}\right)\left(T_{n+1}-T_{n-1}\right)} .
$$

In the same way that $\bar{V}$ is a macroscopic velocity, it is important to understand that $\delta \bar{V} / \delta t$ is a macroscopic acceleration. 


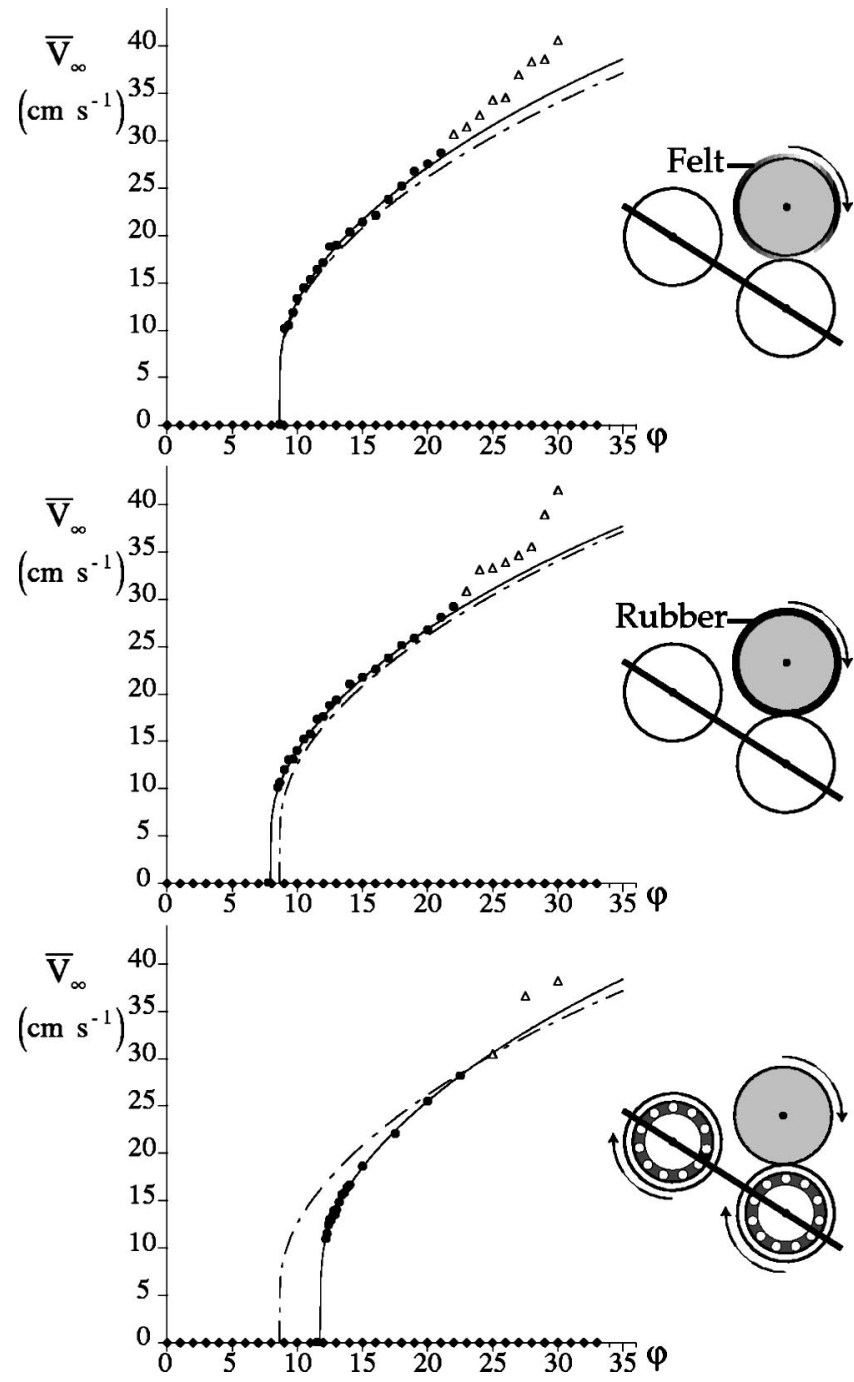

FIG. 5. Same as Fig. 4 but with a roller covered by felt (top), with a roller covered by rubber (middle), and with a brass roller rolling on cylinders that are let free to turn around their axis (bottom). The solid line is the fit by the model and the long-dashed line that obtained for the brass roller (Fig. 4).

It is plotted in Fig. 6 as a function of the velocity for two angles, one below the dynamical angle and the other above. In both cases, the relation appears to be of the form

$$
\frac{\delta \bar{V}}{\delta t} \simeq \gamma(\varphi) g-\kappa(\varphi) \frac{\bar{V}^{2}}{d}
$$

We can see in Fig. 6 that $\kappa(\varphi)$ is related to the curvature and $\gamma(\varphi)$ to the value at the origin. From a physical point of view, $\gamma(\varphi)$ is the effective gravity component along the plane and $\kappa(\varphi)$ is the efficiency of the dissipation by the shocks. It is important to note that the shocks induce macroscopically a force proportional to the velocity square [Eq. (4)] like the turbulent drag and for the same reason. During $\delta t, \bar{V} \delta t / D$ collisions occur, on the average. After each collision, a part of the momentum is lost $(\delta \bar{V} \propto-\bar{V})$ so that globally

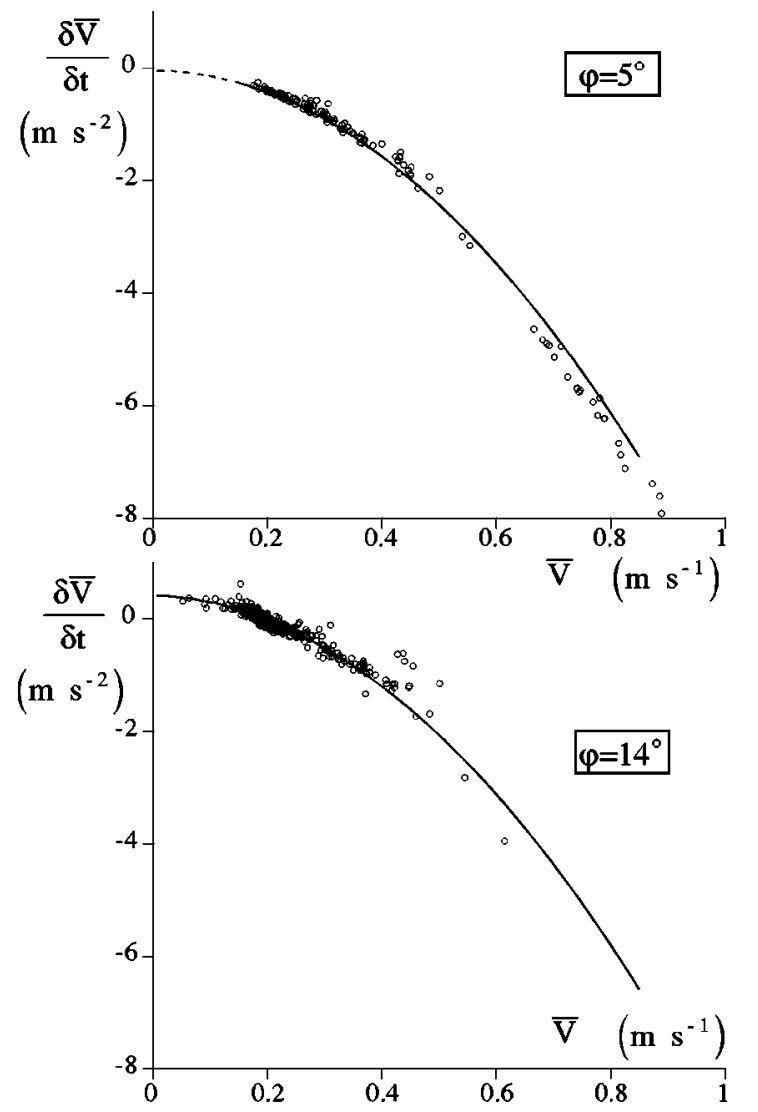

FIG. 6. Acceleration as a function of velocity for two angles: $\varphi=5^{\circ}$, which is below the dynamical angle (top) and $\varphi=14^{\circ}$, which is above the dynamical angle (bottom). The solid line corresponds in each case to the best fit by a quadratic function.

$$
\delta \bar{V} \propto-\frac{\bar{V} \delta t}{D} \bar{V} .
$$

This shows again that the shocks are the main source of energy dissipation.

We measured the two coefficients as functions of the angle $\varphi$ (Fig. 7). $\kappa$ appears to be independent of the angle $\varphi$ and is of order one $(\kappa \simeq 0.21)$. The nondimensional driving

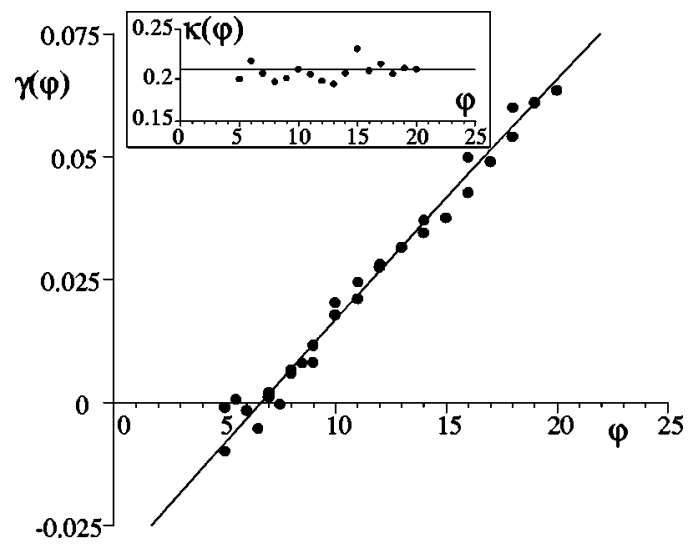

FIG. 7. Main graph: nondimensional driving force $\gamma$ as a function of the angle $\varphi$. Inset: the friction force coefficient $\kappa$ appears to be independent of $\varphi$. 


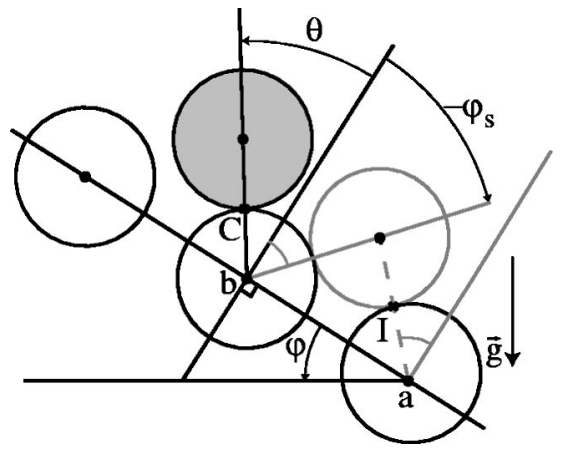

FIG. 8. The roller is assumed to roll on the cylinder $b$ without sliding at the contact point $C$. The position is specified by the angle $\theta$, which varies from $\varphi_{s}$ just after one shock, down to $-\varphi_{s}$, just before the next one.

force $\gamma$ increases with the plane angle and is negative below $6.3^{\circ}$. The experimental points are well fitted by a Coulomblike force of the form

$$
\gamma(\varphi) \simeq \gamma_{\infty}\left(\sin \varphi-\mu_{\infty} \cos \varphi\right)
$$

with $\gamma_{\infty} \simeq 0.27$ and $\mu_{\infty}=\tan \left(6.3^{\circ}\right)$. This formula requires several comments. First, the gravity term (proportional to $g \sin \varphi$ ) had to be corrected by the factor $\gamma_{\infty}$. The apparent gravity is thus smaller than the real one by a factor of 4 . Second, a solidlike friction appears with a critical angle smaller than the dynamical angle (respectively, 6.3 $3^{\circ}$ and $8.5^{\circ}$ ). We will show in the next section that this Coulomb friction term is due to trapping of the roller between the bumps. Globally, the force determined experimentally takes the form

$$
F_{\infty}=\gamma_{\infty} g\left(\sin \varphi-\mu_{\infty} \cos \varphi\right)-\kappa \frac{V^{2}}{d} .
$$

It is unsatisfying in two aspects. First, the equilibrium ( $F$ $=0$ when $V=0)$ does not appear to be a solution. Second, expression (6) would lead to a supercritical bifurcation, the critical slope being $\mu_{\infty}=\tan \varphi_{\infty}$ which is $25 \%$ smaller than the dynamical angle. This means that the hysteresis has disappeared from the macroscopic relation between acceleration and velocity. We will see in the next section how these problems can be bypassed.

\section{MODELING}

\section{A. Discrete modeling}

The modeling of the motion can be simplified by considering that the cylinder rolls without sliding around the cylinder $b$ on the contact point $C$ (Fig. 8). Under this assumption, the energy of the roller,

$$
E=\frac{1+j}{2} m d^{2} \dot{\theta}^{2}+m g d \cos (\theta-\varphi)
$$

is conserved. Consider that the roller has, just after one shock (at the angle $\theta=\varphi_{s}$ ), an angular velocity $\dot{\theta}_{a}$. The angular velocity $\dot{\theta}_{b}$ just before the next shock (at $\theta=-\varphi_{s}$ ) is related to $\dot{\theta}_{a}$ by

$$
\dot{\theta}_{b}^{2}=\dot{\theta}_{a}^{2}+2 \alpha^{2} \sin \varphi_{s} \sin \varphi \frac{g}{d},
$$

where $\alpha$ is related to the ratio of potential energy transferred into translation kinetic energy and is given by

$$
\alpha^{2}=\frac{2}{1+j} \text {. }
$$

The shock between the roller and the next cylinder $a$ dissipates part of the kinetic energy, due to the contact force exerted at the impact point $I$ (Fig. 8). The motion can then be complex, with several rebounds, depending among others on the coefficient of elasticity, but after a time that is small compared to the delay between two shocks, the cylinder rolls again without sliding. If the contact remains linear during the shock, the moment of the contact force about the impact point $I$ should be null. The angular momentum $M$ about the same point $I$ should thus be conserved. Roughly speaking, this means that the rotation around the impact point $I$ does not change during the shock.

Using Fig. 8, the angular momentum $M_{b}$ before the shock can be computed as

$$
M_{b}=\frac{\cos \left(2 \varphi_{s}\right)+j}{2} m d^{2} \dot{\theta}_{b}
$$

Just after the shock, this angular momentum becomes

$$
M_{a}=\frac{1+j}{2} m d^{2} \dot{\theta}_{a} .
$$

The conservation of angular momentum leads to

$$
\dot{\theta}_{a}=\rho \dot{\theta}_{b},
$$

where the restitution coefficient $\rho$ is given by

$$
\rho=\frac{\cos \left(2 \varphi_{s}\right)+j}{1+j} .
$$

The coefficient $\cos \left(2 \varphi_{s}\right)$ corresponds to the conservation of the velocity component tangential to the impact surface. If the distance between the cylinders constituting the plane (and thus $\varphi_{s}$ ) is increased, $\rho$ decreases. The dependence in $j$ reflects the restitution of a part of the rotation kinetic energy into translation motion. $\rho$ thus increases with the moment of inertia $j$.

If we label the successive angular velocities just after the shocks by the index $n$, the recursive relation straightforwardly derives from Eqs. (8) and (10):

$$
\dot{\theta}_{n+1}=\rho \sqrt{\dot{\theta}_{n}^{2}+2 \alpha^{2} \sin \varphi_{s} \sin \varphi \frac{g}{d}} .
$$

It is worth noting that the effective part of the potential energy transformed into translation kinetic energy is $\rho \alpha$, due to the shocks. This is the reason why the apparent gravity $\gamma_{\infty} g$ is smaller than the real one.

Relation (12) is plotted in Fig. 9 and graphically corresponds to a portion of hyperbola. As the restitution coeffi- 


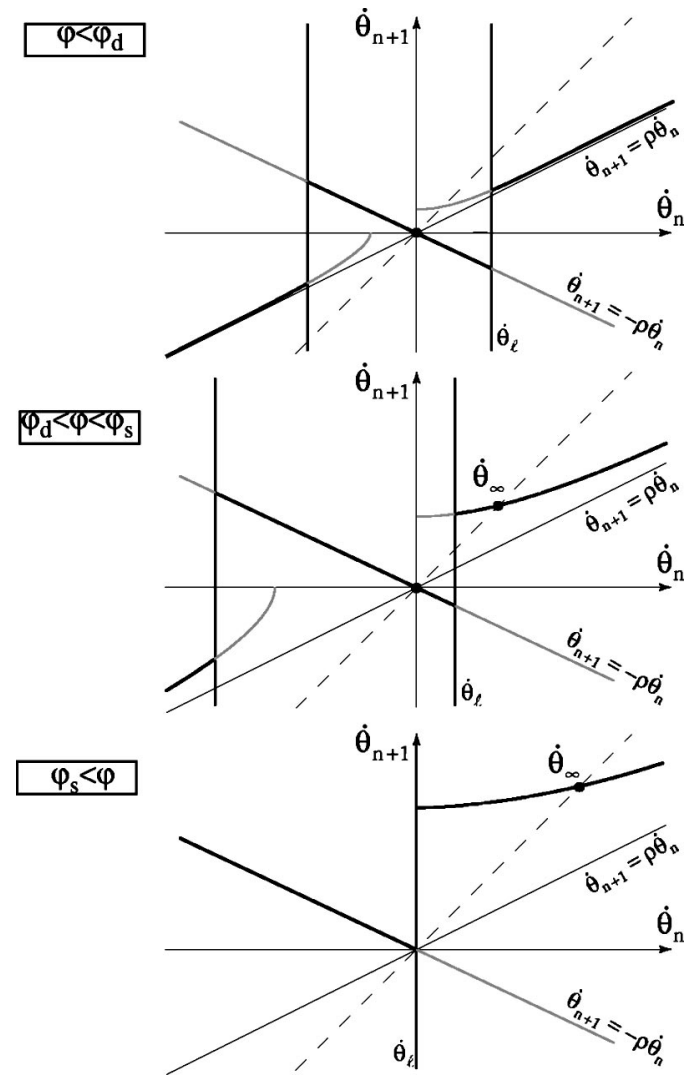

FIG. 9. Relation between the angular velocities just after the shocks $n$ and $n+1$.

cient $\rho$ is smaller than 1 , the recursive relation (12) converges quickly towards its stationary solution $\dot{\theta}_{\infty}$ :

$$
\dot{\theta}_{\infty}=\alpha \rho \sqrt{\frac{2 \sin \varphi_{s}}{1-\rho^{2}}} \sqrt{\frac{g \sin \varphi}{d}} .
$$

This relation simply expresses the balance between gravity and the dissipation by the shocks. $\dot{\theta}_{\infty}$ corresponds on Fig. 9 to the intersection between the hyperbola defined above and the diagonal.

We now have to include a third ingredient. To move from one cylinder to the other, the roller has to escape from the trap, that is to go through the upmost position. We did not consider this condition when we applied the recursive equations to obtain the limit velocity. But if the cylinder, after one shock, does not have enough energy to climb the new cylinder beneath it, it just falls back, and makes a new shock with the previous cylinder, and so on and so forth. The recursive relation is then simply $\dot{\theta}_{n+1}=-\rho \dot{\theta}_{n}$ and the roller converges towards static equilibrium (Fig. 9). This is evidently true only if the trap exists, that is to say if the inclination angle is smaller than the static one. The condition to escape from the trap is that the roller should have enough kinetic energy after the shock to pass over the cylinder. Enough means that the kinetic energy should not become null before $\theta=\varphi$ (see Eq. (7) and Fig. 8). This condition can be written under the form

$$
\dot{\theta}_{n}^{2}>\dot{\theta}_{l}^{2}
$$

where the escape velocity $\dot{\theta}_{l}$ is given by

$$
\dot{\theta}_{l}^{2}=\alpha^{2} \frac{g}{d}\left[1-\cos \left(\varphi_{s}-\varphi\right)\right] .
$$

The limits defined by the potential trapping are represented in Fig. 9 by the vertical-solid lines.

The stationary solution is possible only if the limit angular velocity $\dot{\theta}_{\infty}$ is larger than the threshold $\dot{\theta}_{l}$. This condition defines the dynamical angle:

$$
\frac{1-\cos \left(\varphi_{s}-\varphi_{d}\right)}{2 \sin \varphi_{d} \sin \varphi_{s}}=\frac{\rho^{2}}{1-\rho^{2}}
$$

We are now able to conclude on the origin of the two critical angles. There are mainly three effects: the gravity, the dissipation by the shocks, and the trapping. As in the myth of Sisyphus, the grain rolls up the one beneath it. It then falls down and reaches the bottom of the trap, with a slightly larger velocity coming from gravity. But precisely when its kinetic energy is maximum, a shock occurs that dissipates part of this energy. The balance between the energy brought by the gravity and that dissipated by the shocks determines a limit velocity. But if this limit velocity is smaller than the escape velocity, the roller remains trapped. The dynamical angle is thus the angle for which the limit velocity becomes sufficient to escape from the potential trapping. The static angle is the angle for which the trap disappears.

Starting from the angular velocity $\dot{\theta}_{\infty}$ just after a collision (Eq. 13), the mean velocity $\bar{V}_{\infty}$ reads

$$
\bar{V}_{\infty}=\frac{D}{\int_{-\varphi_{s}}^{\varphi_{s}} \frac{d \theta}{|\theta|}},
$$

where $|\dot{\theta}|$ is related to the angle $\theta$ by

$$
\begin{aligned}
\dot{\theta}^{2}= & \alpha^{2} \frac{g}{d}\left[\left[1-\cos \left(\varphi_{s}-\varphi_{d}\right)\right] \frac{\sin \varphi}{\sin \varphi_{d}}+\cos \left(\varphi_{s}-\varphi\right)\right. \\
& -\cos (\theta-\varphi)] .
\end{aligned}
$$

The limit mean velocity [Eq. (17)] can be computed either using the Legendre elliptical integral of first kind [11] or numerically. The resulting curve is shown on Fig. 10 for $\varphi_{d}=8.5^{\circ}$ and $\varphi_{s}=33^{\circ}$. The equilibrium (case 1 in Fig. 3) is possible for $\varphi<\varphi_{s}$, and corresponds to a null mean velocity. For the periodic motion (case 3 in Fig. 3), the mean velocity increases very quickly above the threshold $\varphi_{d}$. Indeed, it is easy to show that it is approximately equal to

$$
\bar{V}_{\infty} \simeq-\frac{\sqrt{2 \alpha g d}}{\ln \left(\varphi-\varphi_{d}\right)}
$$

for small $\varphi-\varphi_{d}$. It is worth noting that for this particular bifurcation the order parameter does not scale as a power law but as the inverse of a logarithm. But this is not the only particularity of this bifurcation. The two stable stationary states are separated one from the other by an unstable solution: the equilibrium of the roller on the top of the cylinder 


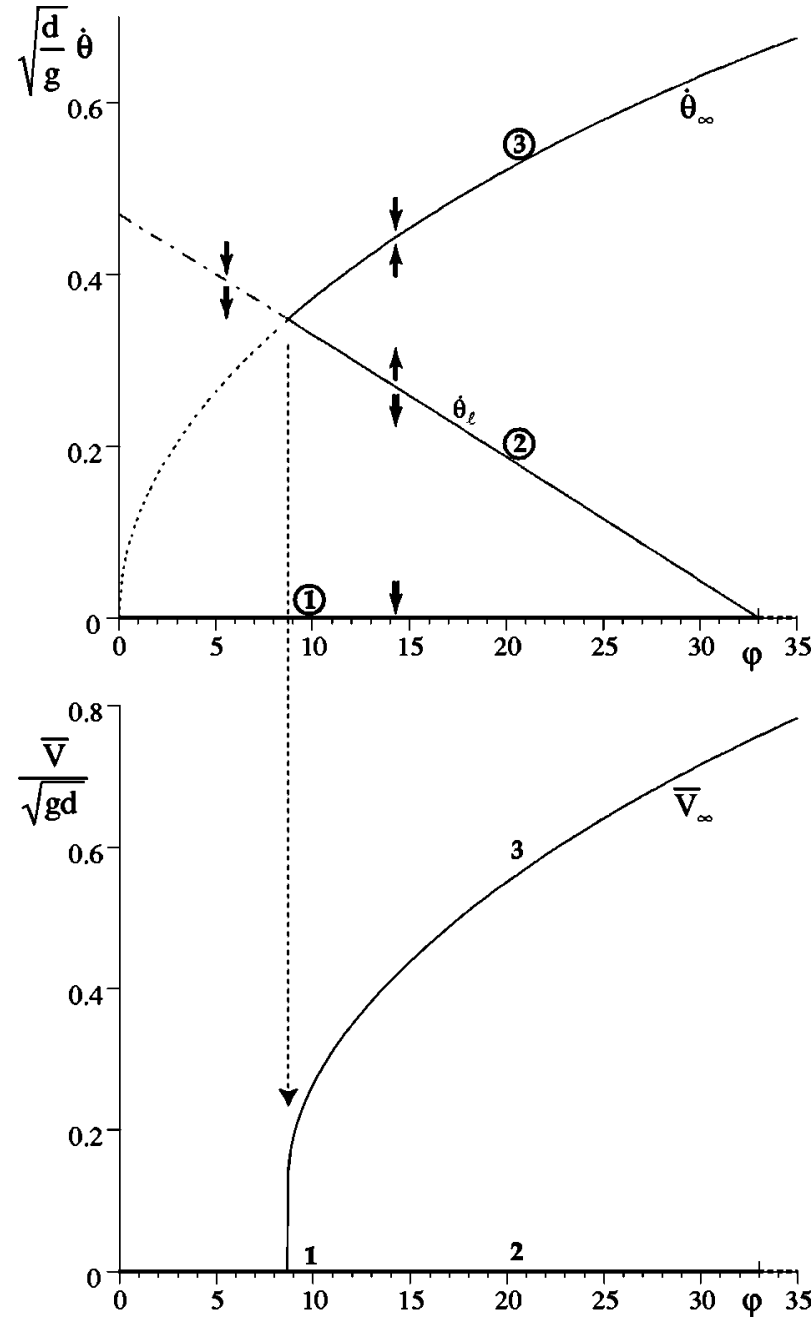

FIG. 10. This graph shows the three possible stationary states (see Fig. 3), both for the microscopic angular velocity $\dot{\theta}$ and for the local mean velocity $\bar{V}$ : (1) the static equilibrium $\left(\dot{\theta}_{\infty}=0\right.$ and $\bar{V}_{\infty}$ $=0$ ) for $\varphi<\varphi_{s}$, (2) the condition after one shock leading just to the unstable equilibrium on the top of the cylinder underneath $\left(\dot{\theta}=\dot{\theta}_{l}\right.$ $>0$ but $\bar{V}_{\infty}=0$ ) for $\varphi_{d}<\varphi<\varphi_{s}$, and (3) the periodic motion ( $\dot{\theta}_{\infty}$ $>0$ and $\bar{V}_{\infty}>0$ ), which is reached only for $\varphi>\varphi_{d}$. If $\dot{\theta}$ is smaller than $\dot{\theta}_{l}$ the roller quickly goes back to equilibrium. If above, it converges toward the constant velocity regime.

below it (case 2 in Fig. 3). To reach this equilibrium, one has to give the roller an initial angular velocity $\dot{\theta}_{\infty}$ equal to the escape velocity $\dot{\theta}_{l}$. The roller then reaches its equilibrium position infinitely slowly. The corresponding mean velocity $\bar{V}_{\infty}$ is thus null and the unstable branch collapses with the metastable one (noted 2 and 1 on Fig. 10, bottom). This singular behavior comes from the fact that $\bar{V}_{\infty}$ is a macroscopic velocity: it can be null even when the roller is not at rest but oscillates in the potential trap.

\section{B. Comparison with experimental results}

We have fitted the experimental data by adjusting the two physical parameters of the model: the restitution coefficient $\rho$ and the effective part of potential energy restituted into translation kinetic energy $\rho \alpha$. The resulting curves are shown on
Fig. 4 for the brass cylinder and in Fig. 5 for the roller covered by felt and by rubber. In the three cases, $\rho \alpha$ is found to be very close to the expected value (respectively, 0.69 , 0.70 , and 0.71 instead of 0.71 ). The restitution coefficient $\rho$ is $10 \%$ smaller than its predicted value $(0.59,0.59$, and 0.62 instead of 0.66). The small difference between the three results could be mainly due to the slight increase of the roller radius, when covered by rubber. As a conclusion, the dynamics of the real system is completely determined by the three effects taken into account in the model: driven by the gravity, dissipation by the shocks, and potential trapping.

To investigate the large-angle regime, we can add a fourth effect: when the centrifugal force $m \dot{\theta}^{2} d$ becomes larger than the radial component of the gravity force $m g \cos (\theta-\varphi)$, the roller takes off. It then makes a parabolic free flight before colliding with the rough plane. We can assume that the shock is instantaneous so that the momentum of inertia about the collision point is conserved. We integrated numerically the equation of motion with this further effect. We then observed that the limit mean velocity $\bar{V}_{\infty}$ is essentially the same for angles $\varphi$ smaller than $21^{\circ}$ and then increases (solid line in Fig. 4). Indeed, after a free flight the impact parameter is smaller than previously so that the restitution of energy is larger. Above a threshold angle $\varphi \simeq 27.1^{\circ}$, the constant velocity regime disappears and the roller indefinitely accelerates. This is due to the fact that the free flights becomes longer and longer as the velocity increases so that the number of shocks per unit time becomes smaller and smaller. These two behaviors are observed in the experiment (Fig. 4).

In the case where the cylinders composing the rough plane are let free to turn around their axis (Fig. 5, bottom), there still exists some values of the two parameters $\rho \alpha$ and $\rho$ for which the fit is good. The effective part of potential energy transferred into translation kinetic energy $\rho \alpha$ is again 0.71 . But evidently, the restitution coefficient is strongly decreased by this change $(\rho=0.48)$.

\section{Continuous modeling}

The conclusion of the previous sections is that considering the mean velocity $\bar{V}$ leads one to forget one of the main aspects of the dynamics: the subcritical transition between equilibrium and motion, or in other words the stability of the static state. This comes from the fact that macroscopically, we do not want to know anything about the microscopic position of the roller with respect to the plane bumps. To correct the force $F_{\infty}$ derived from the experimental measurements, we have to introduce a matching between two velocities. At large velocity, $V$ has to be the mean velocity $\bar{V}$ but at small velocity, $V$ has to describe the motion of the roller in the potential trap $(d \dot{\theta})$. This lead us to introduce a matching between the force $F_{\infty}$ previously determined and a trapping force $F_{0}$. This can be done in the following way:

$$
\frac{d V}{d t}=(1-h) F_{\infty}+h F_{0}
$$

where the matching function $h$ should tend to 1 when the velocity $V$ tends to 0 and should tend to 0 when the velocity is much larger than the escape velocity $d \dot{\theta}_{l}$. 

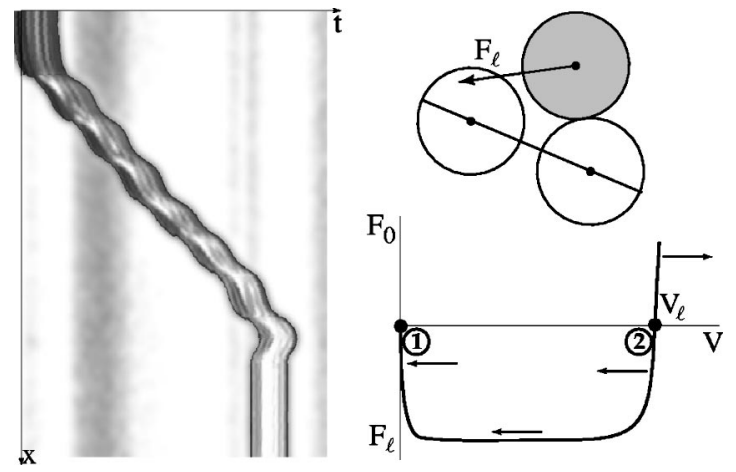

FIG. 11. Left: spatiotemporal trajectory for an angle $\varphi$ just below the dynamical angle. After a short transient, the roller is trapped. Right: when the roller is trapped (for small initial velocity $V$ ), it is submitted to a force $F_{0} \simeq F_{l}$, which brings it back to equilibrium $(F=0$ for $V=0)$. When the velocity $V$ (at the bottom of the trap) is sufficient to escape from the trap $\left(V>V_{l}\right)$, the force $F$ suddenly becomes positive.

For small velocity, we thus assume that the roller is trapped (Fig. 11). Small velocity means that the initial kinetic energy is not sufficient to escape from the trap, i.e., that the velocity $V$ is smaller than the escape velocity $V_{l}$ :

$$
V_{l} \propto \sqrt{g d\left[1-\cos \left(\varphi-\varphi_{s}\right)\right]} .
$$

It is then submitted to the trapping force $F_{l}$

$$
F_{l} \propto g \sin \left(\varphi-\varphi_{s}\right)
$$

which brings the roller back to equilibrium. That implies the fact that $F$ should be null when the velocity $V$ vanishes. These basic properties are summarized on Fig. 11 and constrain the construction of the microscopic force $F_{0}$.

As we have seen, the force $F_{0}$ tends to 0 when the trap disappears. It is the case if we assume the proportionality to $g\left(\sin \varphi-\mu_{s} \cos \varphi\right)$ which is simply the development of expression 21. To introduce the equilibrium, we can assume a force proportional to $\sqrt{V}$, which interestingly leads to an abrupt stop, in finite time and length. With these two constraints, $F_{0}$ reads

$$
F_{0}=\gamma_{0} \sqrt{\frac{V}{V_{0}}} g\left(\sin \varphi-\mu_{s} \cos \varphi\right),
$$

where $V_{0}$ is a typical escape velocity (a fraction of $\sqrt{g d}$ ) and $\gamma_{0}$ is a nondimensional prefactor of the order of unity.

Finally, it can be seen in Eq. (20) that the escape velocity depends linearly on the slope near the static angle $\varphi_{s}$. It is easy to show that this implies that $(1-h)$ should be proportional to $V^{3 / 2}$ for small $V$ such as for instance: $h=\exp [$ $\left.-\left(V / V_{0}\right)^{3 / 2}\right]$.

The parameters $V_{0}$ and $\gamma_{0}$ were adjusted to recover the correct dynamical angle $\varphi_{d}\left(V_{0} \simeq 0.12 \sqrt{g d}\right.$ and $\left.\gamma_{0} \simeq 0.12\right)$. The force is plotted on Fig. 12 as a function of velocity for several angles. For large velocities, the force depends quadratically on velocity, as previously, but the force is now null for $V=0$ and negative for small velocities, as required (see Fig. 11). We observe that the dynamical angle corresponds to

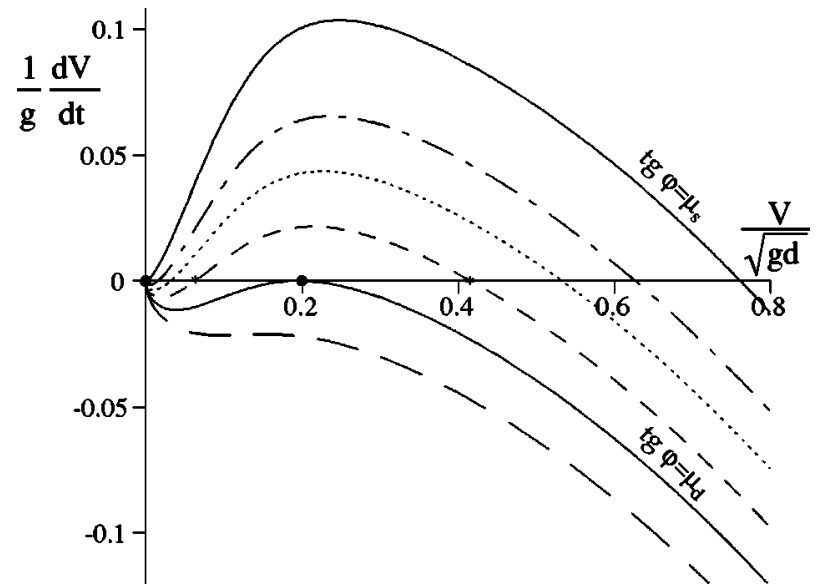

FIG. 12. Continuous model of the force globally acting on the roller. The force is plotted as a function of velocity for different angles: $\varphi=4^{\circ}$ (long-dashed line), $\varphi=\varphi_{d}$ (solid line), $\varphi=14^{\circ}$ (dashed line), $\varphi=19^{\circ}$ (dotted line), $\varphi=24^{\circ}$ (dotted-dashed line), and $\varphi=\varphi_{s}$ (solid line).

the angle for which a new stationary state $(F=0)$ appears and that the static angle corresponds to the angle at which the trap disappears.

The stationary states obtained are plotted in Fig. 13 (dashed line). The experimental data are still fitted by the continuous model but the subcritical character of the transition between equilibrium and flowing is now recovered in this macroscopic description, without introducing any knowledge of the microscopic position. If one is interested by the description of the large-angle regime, where the roller accelerates indefinitely above a threshold angle, it is sufficient to let the force $F_{\infty}$ tend towards a constant Coulomb friction at large velocities. The description of the motion of one grain on a rough plane is then complete (Fig. 13, solid line).

\section{CONCLUDING REMARKS}

This experiment is only an outline of real grains motion on a sand pile. First because it is two dimensional, second

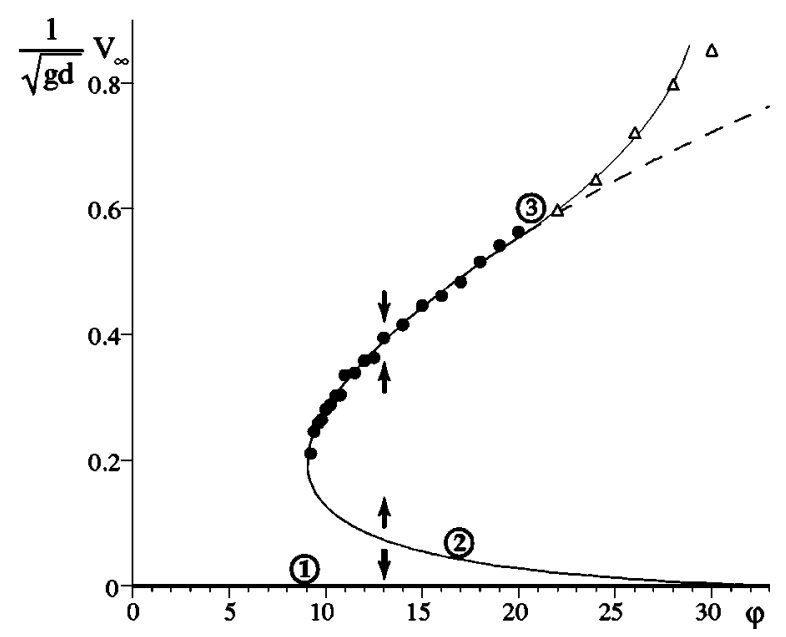

FIG. 13. Stationary states in the continuous model of the force globally acting on the roller. The possible limit velocities are plotted versus the angle. 
because the geometry is regular, and third because the underneath grains can also start moving in real sand piles. However, we think that the most important mechanisms are captured and that the differences essentially lead to change the values of the critical angles $\varphi_{d}$ and $\varphi_{s}$. In particular, the simple experiment proposed in this paper explains the origin of the hysteresis commonly observed in granular media between flowing and static states and the constant velocity achieved in the flowing regime. The velocity comes from the balance between the gain in kinetic energy due to gravity and its loss during shocks. The hysteresis comes from the fact that a grain has always to pass above underneath grains. If it has enough energy, it flows, but if not, it remains trapped. On the other hand, if the angle is too large, the grain accelerates indefinitely as it jumps above the grains, gains more kinetic energy, and dissipates less due to fewer shocks.

The simplicity of this experiment also allows us to compute all the details of the dynamics. We find that the bifurcation has a particularity that comes from the fact that we have to go from a microscopic description, the angular motion of one cylinder above the underneath one, to a macroscopic description of the average velocity. It is a degenerated subcritical bifurcation in which the metastable and unstable branches are collapsed. Correspondingly, the order parameter does not scale as a power law just above the threshold but as the inverse of a logarithm. In the basic macroscopic approach, we thus loose the description of the metastable equilibrium in the traps formed by the plane bumps, giving the subcritical bifurcation an unusual aspect.

With this experiment it is possible to investigate not only the stationary regime but also the transients (acceleration), and thus to measure the effective macroscopic force acting on the roller. We find that the effective gravity is reduced and that a Coulomb friction force naturally appears. But to really build a force model we have to reintroduce the hysteretic behavior by making a matching for small velocity to the microscopic trapping. This description of the force has then the advantage of being well (continuously) defined in the whole range of velocities (it can thus be used in numerical simulations), but also of reproducing interesting properties (observed) such as a stop in finite time and length, and an acceleration divergence for large angles. What remains to be done is to investigate the effect of the same three mechanisms (gravity, dissipation by shocks, and potential trapping) in more complex situations and to see if the force built here can be adapted to model granular motion in real sandpiles [12].
[1] C. Coulomb, Memoires de Mathmatiques et de Physique Presents l'Academie Royale des Sciences par Divers Savants et Lus dans les Assembles (Imprimerie Royale, Paris, 1776), p. 343.

[2] A. Daerr and S. Douady, Europhys. Lett. 47, 324 (1999).

[3] G. H. Ristow, F. X. Riguidel, and D. Bideau, J. Phys. I 4, 1161 (1994).

[4] F. X. Riguidel, R. Jullien, G. H. Ristow, A. Hansen, and D. Bideau, J. Phys. I. 4, 261 (1994).

[5] D. Bideau, I. Ippolito, L. Samson, G. G. Batrouni, S. Dippel, A. Aguirre, A. Calvo, and C. Henrique, in Proceedings of the Workshop on Traffic and Granular Flow, edited by D. E. Wolfer, M. Schreckenberg, and A. Bachem (World Scientific, Singapore, 1996), p. 279.

[6] G. G. Batrouni, S. Dippel, and L. Samson, Phys. Rev. E 53,
6496 (1996).

[7] S. Dippel, G. G. Batrouni, and D. E. Wolf, Phys. Rev. E 54, 6845 (1996).

[8] A. Aguirre, I. Ippolito, A. Calvo, C. Henrique, and D. Bideau, Powder Technol. 92, 75 (1997).

[9] L. Samson, I. Ippolito, S. Dippel, and G. G. Batrouni, Powders \& Grains 97, 503 (1997).

[10] C. D. Jan, H. W. Shen, C. H. Ling, and C. L. Chen, in Proceedings of the Ninth Conference On Engineering Mechanics, College Station, Texas, 1992, edited by L. D. Lutes and J. M. Niedzwecki (American Society of Civil Engineers, New York, 1972), p. 768.

[11] C. Ancey, P. Evesque and P. Coussot, J. Phys. I 6, 725 (1996).

[12] S. Douady, B. Andreotti, and A. Daerr, Eur. Phys. J. B 11, 131 (1999). 